\title{
Simulation of Fluorescent Concentrators
}

\author{
Marion Bendig * \\ Ulm University
}

\author{
Holger Dammertz $\ddagger$ \\ Ulm University \\ Michael Weber $\|$ \\ Ulm University \\ Jan Christoph Goldschmidt ${ }^{\S}$ \\ Fraunhofer ISE
}

\begin{abstract}
Fluorescent concentrators (see Figure 1) are developed and investigated in solar energy research in order to improve the efficiency and applicability of solar cells. This is achieved by concentrating even diffuse light on cloudy days on small solar cells, using a fluorescent dye enclosed in low-cost acrylic glass.

We developed a Monte Carlo simulation model for fluorescent concentrators that makes it possible to test and improve different setups and to gain further insight into the physical processes.

As it is a complicated topic, fluorescent light has been investigated very rarely in computer graphics. Recent advances in fast ray tracing give the opportunity to simulate complex physical processes using simple models. Changes of the setup and physical properties can be simulated and evaluated quickly, providing the necessary foundation for automatic optimization.
\end{abstract}

Index Terms: I.3.3 [Computing Methodologies]: Computer Graphics-Picture/Image Generation; G.3 [Mathematics of Computing]: Probability and Statistics-Monte Carlo; J.2 [Computer Applications]: Physical Sciences and Engineering-Physics

\section{INTRODUCTION}

Photovoltaic systems are designed to reduce the need for nuclear power or fossil fuels. Fluorescent concentrators have been developed to enhance the production of energy via solar cells and to reduce costs for this. They can concentrate both direct and diffuse light, which increases the efficiency of solar cells especially in cloudy weather.

A fluorescent concentrator is made from PMMA (acrylic glass) and contains a dye. Figure 2 illustrates the basic setup for one application of a fluorescent concentrator: If light enters the concentrator and hits a dye molecule it will be absorbed and re-emitted at a different wavelength according to the photoluminescence spectrum (PL-spectrum).

Since the PL-spectrum is shifted to longer wavelengths as compared to the absorption spectrum (see Figure 3), light is unlikely to be re-absorbed and therefore travels through the medium mostly undisturbed. By designing the fluorescent concentrator in a certain shape, light is trapped inside and can be guided to the solar cell due to total internal reflection.

In spite of the advantages this concept provides, the research interest almost vanished for a long time, because the expected efficiency was not reached. In the last few years new ideas and materials have been developed and the potential of fluorescent concentrators is now reinvestigated. The testing of new concepts in

\footnotetext{
*e-mail: marion.bendig@uni-ulm.de

†e-mail: johannes.hanika@uni-ulm.de

‡e-mail: holger.dammertz@uni-ulm.de

$\S$ e-mail: jan.christoph.goldschmidt@ise.fraunhofer.de

ฯe-mail: marius.peters@ise.fraunhofer.de

|le-mail:michael.weber@uni-ulm.de
}

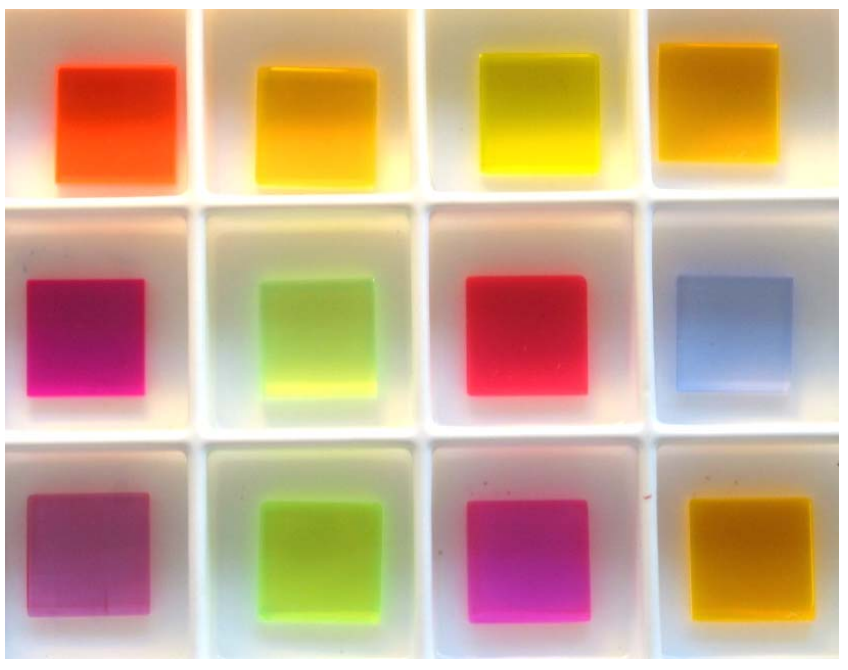

Figure 1: Photograph of the fluorescent concentrator probes which are used for the measurements in this paper.

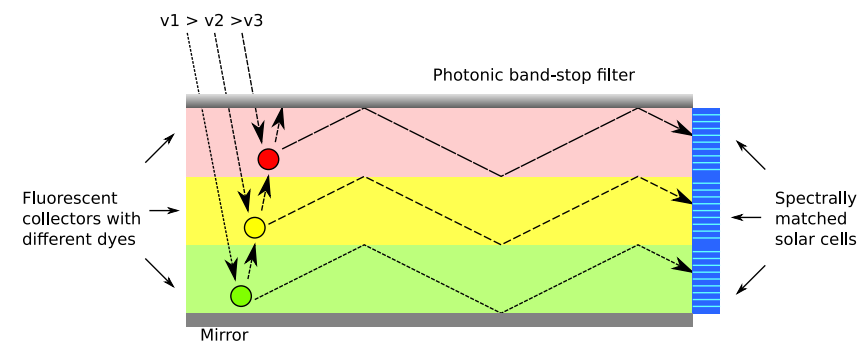

Figure 2: Concept of a fluorescent concentrator: the absorbed light is re-emitted at a different wavelength in the dye and then guided to the solar cell, with a very low probability of re-absorption. One can improve this by using a concentrator stack, as shown in the figure.

experiments, however, is difficult and expensive. Not all physical processes in a fluorescent concentrator are fully understood yet. For analytical calculation and simulation they are far too complex.

Through Monte Carlo simulation, it is possible to check the existing physical models of fluorescent concentrators and to thoroughly test new ideas. Thus enhanced concepts can be invented and proved before their realization. The data and techniques acquired during our investigations can also be applied to Monte Carlo ray tracing applications to simulate fluorescence in the context of computer graphics.

\section{Related Work}

There are several publications about simulation (and even Monte Carlo simulation) of fluorescent light in various fields of science, for example by Welch et al. [16], and Susila and Naus [15]. In computer graphics very little research in this domain has been 


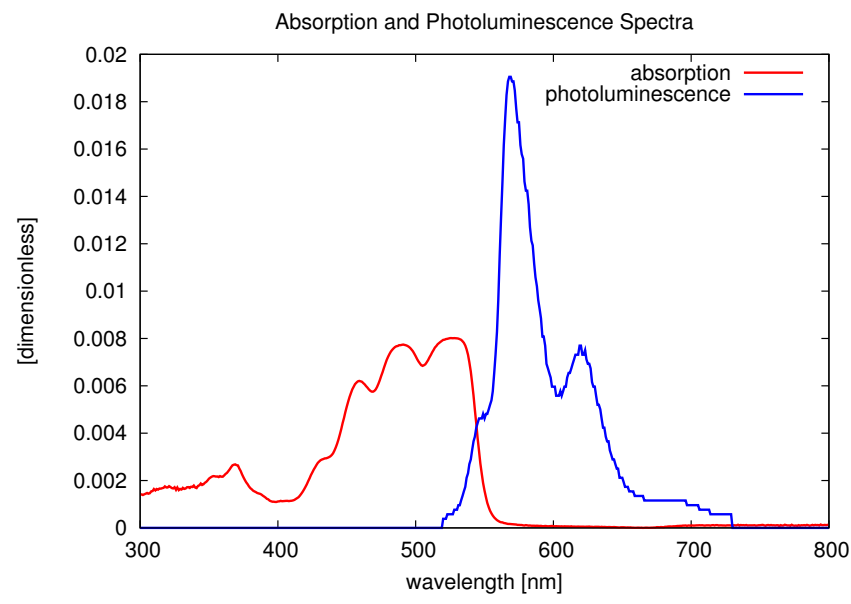

Figure 3: Fraction of overall absorption events occurring at a certain wavelength (absorption spectrum) and fraction of light re-emitted at a certain wavelength (photoluminescence spectrum, PL) for the fluorescent dye used in this paper.

done. Glassner [8] presented a formulation of the rendering equation including phosphorescence and fluorescence, i.e., a mathematical model for global energy balancing which includes these phenomena. After his work, apparently there has not been any further investigation of fluorescence phenomena in computer graphics until 2001. Wilkie et al. implemented a rendering system including fluorescence and polarization using Stokes vectors and Müller matrices [17]. In 2006 they provided an analytical BRDF model for fluorescent surfaces [18], but this approach only works for a finite set of wavelengths.

Our work is based on the dissertation of Zastrow [19] and the publications of Peters, Goldschmidt et al. [9, 12] about fluorescent concentrators. It focuses on the physically correct simulation of fluorescent concentrators in order to gain deeper knowledge about the physical processes involved and to optimize the concentrator.

Heidler [10] developed a Monte Carlo model for fluorescent concentrators in 1982. His model was made for an efficiency analysis for the concentrator. Carrascosa et al. [4] were first to describe ray tracing of fluorescent concentrators.

In the last few years Burgers et al. [3, 2] and Schüler et al. [13] used Monte Carlo ray tracing for simulations of fluorescent concentrators and quantum dot solar concentrators, respectively.

We conduct additional experiments, as e.g. the angular experiment and use advances in fast ray tracing [5] research which make a quick simulation possible and even allow for interactive previews for dynamically changing material parameters.

\section{Monte Carlo Simulation of fluorescent Con- CENTRATORS}

\subsection{Fluorescent Concentrators}

The efficiency of fluorescent concentrators depends on several aspects. Figure 4 illustrates important internal processes of the concentrator. There are quite a few interactions that result in loss of energy.

First of all, due to reflection, not all the light that hits the concentrator enters the medium. Light in the medium can be absorbed in the PMMA or a dye molecule. When the light hits the dye it can be absorbed and may be re-emitted at a longer wavelength, resulting in energy loss. The distribution of the re-emitted wavelengths is described by the photoluminescence (PL-) spectrum. The probability of absorption is wavelength dependent and described by the absorp- tion spectrum. As Figure 3 illustrates, the PL spectrum is shifted to the longer wavelengths compared to the absorption spectrum, but the two spectra overlap. If a photon is re-emitted with a wavelength in the overlap it can be re-absorbed in the dye. This leads to additional energy loss. Moreover not all absorbed light is re-emitted, depending on the quantum efficiency of the dye.

Light can leave the concentrator when it hits the boundary in a steep angle (loss cone). Even if the angle is bigger than the critical one it may not be totally reflected because of imperfections in the concentrator material.

External factors of the efficiency of a fluorescent concentrator are the shape, dimensions and concentration of the dye in the PMMA. Additionally filters and diffuse reflectors can be attached to the boundary. Finally the efficiency depends on the direction and spectral distribution of the incoming light.

\subsection{Simulation Model}

Parts of the processes in a fluorescent concentrator are well known and can be described by analytical models. But for example the behavior of the dye is still not fully understood and difficult to measure in an experimental setup. In order to build a simulation we have to rely on existing models and experimental data. The required input parameters are reconstructed from the measured absorption and photoluminescence spectrum.

We used a range from 300 to 800 nanometers of the AM 1.5 spectrum [1] for the input wavelengths. The dye in the concentrator we used to test our model was BA241. This data can easily be replaced to simulate different conditions, for instance a different dye.

\subsubsection{Model Development}

We decided to simulate single particles (photons) with one wavelength instead of weighted paths. These particles are traced on their way through the fluorescent concentrator. Each of the interactions described in Section 3.1 is modelled as independent event. This approach requires more rays to be shot than other methods which transport differential energy per path, but on the other hand it is less error prone in the implementation and numerically more robust.

The concentrator material is assumed to be a homogeneous medium, consisting of PMMA and a fluorescent dye. In this medium a photon can experience different events, such as reflection and refraction at the boundary, absorption, scattering and fluorescence (see Figure 4).

Due to incomplete data we had to simplify the initial model. As a first approximation to the real conditions we assume the phase function of the dye to be isotropic, the quantum efficiency to be wavelength independent and the PL-spectrum to be independent of the incoming wavelength. The concentrator material is assumed to be a homogeneous medium, consisting of PMMA and a fluorescent dye. We disregard the polarization of the light, irregularities of the material and some rare events that can happen in the concentrator. Later we will add more ameliorations to our model.

\subsubsection{A Photon's Path through the Fluorescent Concentra- tor}

This section details the events occurring in the probe, as illustrated in Figure 4. As a ray hits the boundary of the fluorescent concentrator we calculate the reflectivity using the Fresnel equations [6]:

$$
\begin{aligned}
R_{s} & =\left(\frac{\sin \left(\alpha_{\text {in }}-\alpha_{\text {out }}\right)}{\sin \left(\alpha_{\text {in }}+\alpha_{\text {out }}\right)}\right)^{2} \\
& =\left(\frac{n_{1} \cos \left(\alpha_{\text {in }}\right)-n_{2} \cos \left(\alpha_{\text {out }}\right)}{n_{1} \cos \left(\alpha_{\text {in }}\right)+n_{2} \cos \left(\alpha_{\text {out }}\right)}\right)^{2} \\
R_{p} & =\left(\frac{\tan \left(\alpha_{\text {in }}-\alpha_{\text {out }}\right)}{\tan \left(\alpha_{\text {in }}+\alpha_{\text {out }}\right)}\right)^{2}
\end{aligned}
$$




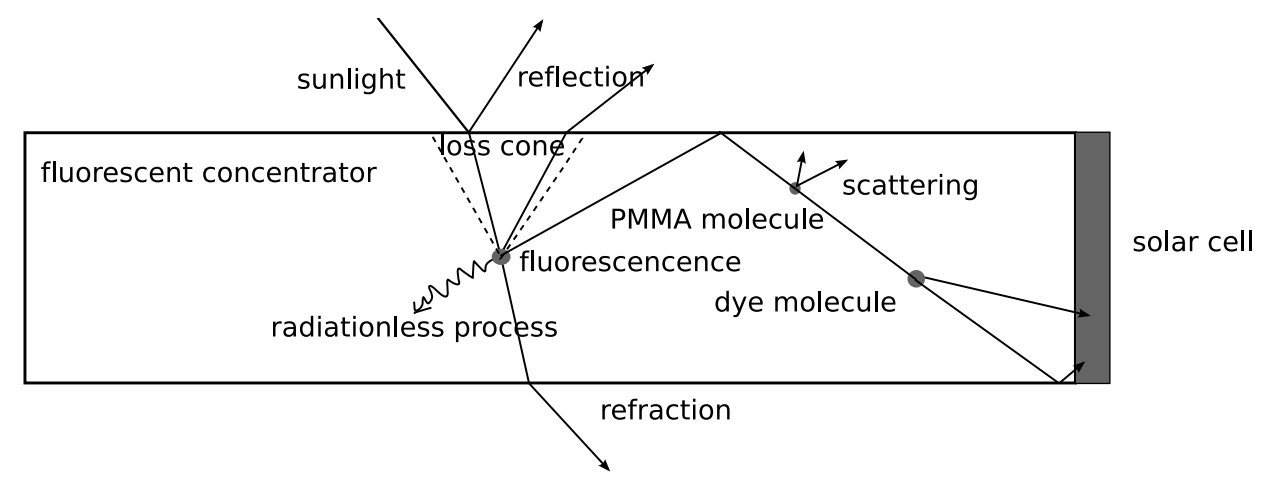

Figure 4: Processes in a fluorescent concentrator.

$$
\begin{aligned}
& =\left(\frac{n_{2} \cos \left(\alpha_{\text {in }}\right)-n_{1} \cos \left(\alpha_{\text {out }}\right)}{n_{2} \cos \left(\alpha_{\text {in }}\right)+n_{1} \cos \left(\alpha_{\text {out }}\right)}\right)^{2} \\
R & =\frac{R_{s}+R_{p}}{2},
\end{aligned}
$$

where $R_{s}$ and $R_{p}$ denote the reflectivity of the perpendicular and the parallel component of the light, respectively. As we assume the light to be unpolarized, it is appropriate to use the average $R$ as reflectivity in the simulation. $\alpha_{\text {in }}$ is the incident angle and $\alpha_{\text {out }}$ the angle of refraction. $n_{1}$ is the refractive index of the medium from which the ray comes and $n_{2}$ the refractive index of the target medium. These are calculated depending on the wavelength using the two-term form of the Cauchy equation:

$$
n(\lambda)=c_{1}+\frac{c_{2}}{\lambda^{2}},
$$

where $c_{1}$ and $c_{2}$ are material-specific coefficients and $\lambda$ is the wavelength. The angle of refraction is calculated according to Snell's law:

$$
\frac{\sin \left(\alpha_{\text {in }}\right)}{\sin \left(\alpha_{\text {out }}\right)}=\frac{n_{2}}{n_{1}}
$$

The reflectivity gives us the probability of a reflection event for the Monte Carlo simulation. If after the reflection or refraction event the ray is (still) in the concentrator, we calculate the new ray direction according to the event.

Then the path length $\Delta z$ of the ray is calculated depending on the wavelength using the Lambert-Beer law and the Monte Carlo inversion method $[7,14]$ :

$$
\Delta z=-\frac{\log (1-\xi)}{\alpha_{\text {total }}(\lambda)}
$$

where $\alpha_{\text {total }}$ is the sum of the absorption coefficients of the dye, $\alpha_{d y e}$, and the PMMA, $\alpha_{p m m a}$, which are deduced from the measured absorption data. $\xi$ is sampled randomly uniformly distributed from $[0,1)$. According to the path length the next event is either an interaction with the boundary again or an absorption in the dye or PMMA. When the ray is absorbed before it hits the boundary the probability of the event to be a dye absorption is $\frac{\alpha_{d y e}}{\alpha_{\text {total }}}$ and to be a PMMA absorption accordingly $\frac{\alpha_{p m m a}}{\alpha_{\text {total }}}$. If the ray is absorbed in the dye, the chance of a fluorescence event is given by the quantum efficiency $Q E$. For the dyes that we use it is normally approximately $95 \%$, depending on the dye. In case of a fluorescence event, a new wavelength is sampled from the photoluminescence spectrum and a new direction is sampled. Currently, we use an isotropic phase function.

After this a new path length is sampled and the process repeats.

During the simulation all kinds of information, as number, kind and location of events, path length etc., can be collected. This information is evaluated at the end of each path to generate statistics.

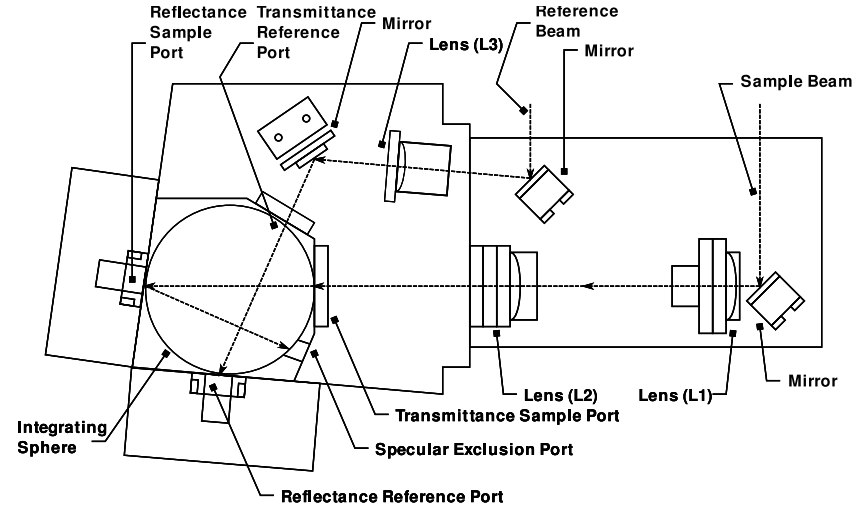

Figure 5: Experimental setup for the measurement of various properties of fluorescent concentrator probes.
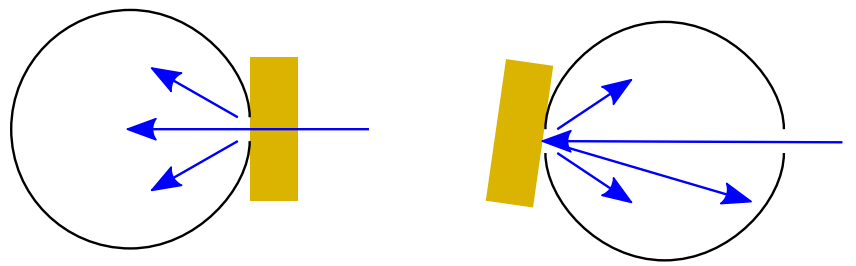

Figure 6: Measurement of the transmission (left) and reflection (right) component in a so called Ullbricht sphere.

\section{Results}

\subsection{Model Verification}

To evaluate and improve the correctness of our simulation model we reproduced a couple of measurements that were made with real fluorescent concentrators. Amongst others we determined the absorption and the reflection of the concentrator through simulation. The real measurements were made using a photospectrometer and an integrating sphere (see Figure 5). The challenge of the experimental measurement is the adjustment of the apparatus and the determination of the desired quantity in spite of measurement errors.

For a comparison with the measured data it is necessary to fit the simulation process as best as possible to the experiment.

Transmission Experiment This experiment captures all light which passes through the probe without absorption event and without being reflected at the top. The probe is attached in the apparatus (Figure 5) as shown in Figure 6 (left). This way, the transmittance of the material can be measured. The Ullbricht sphere is used to 


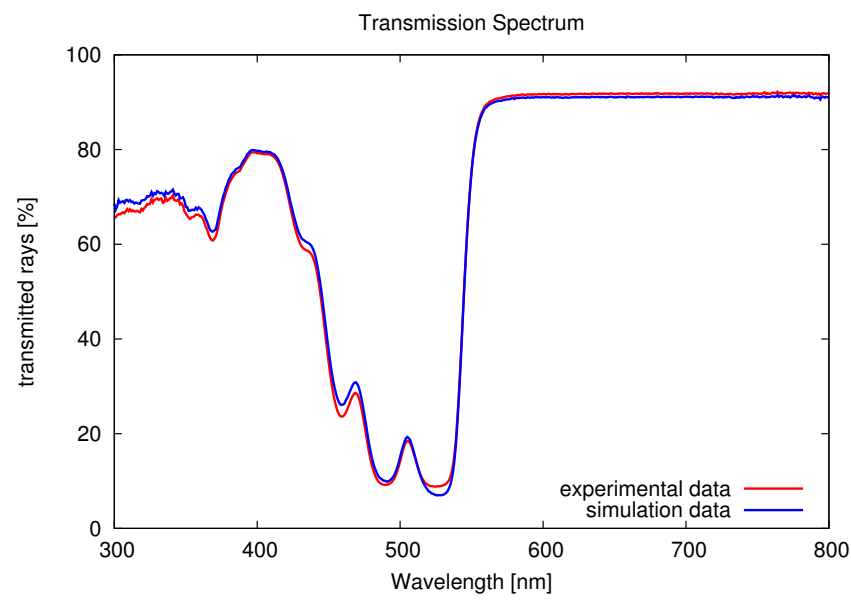

Figure 7: Results for the transmission experiment. The graph shows data from our simulation in comparison to the measured data.

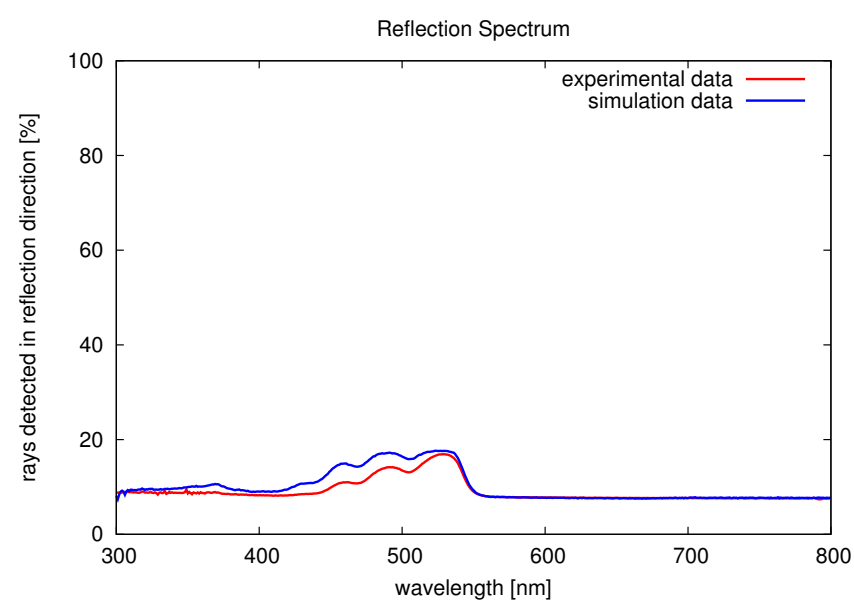

Figure 8: Comparison of the data from our simulation and the measured data for the reflection experiment.

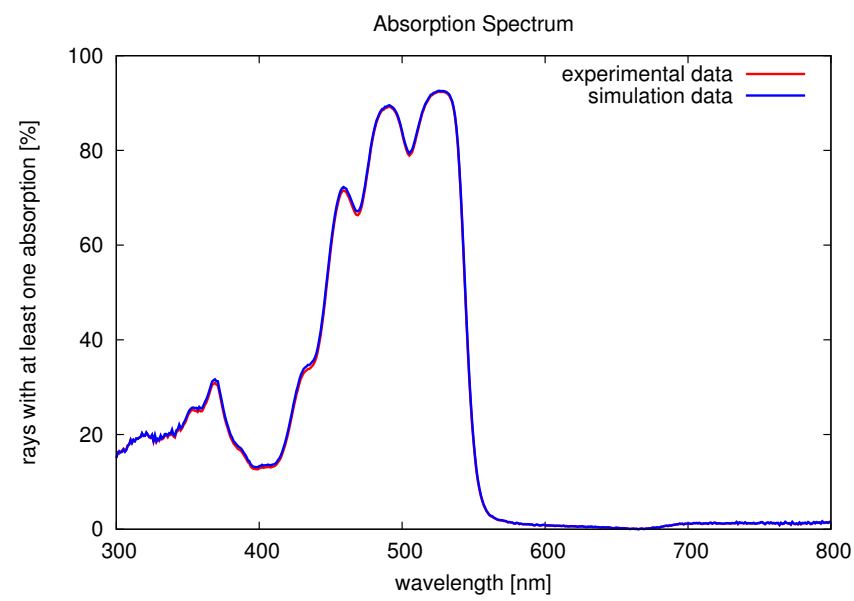

Figure 9: Results for the absorption experiment. The graph shows data from our simulation in comparison to the data that was calculated using the data from the transmission and the reflection experiments.

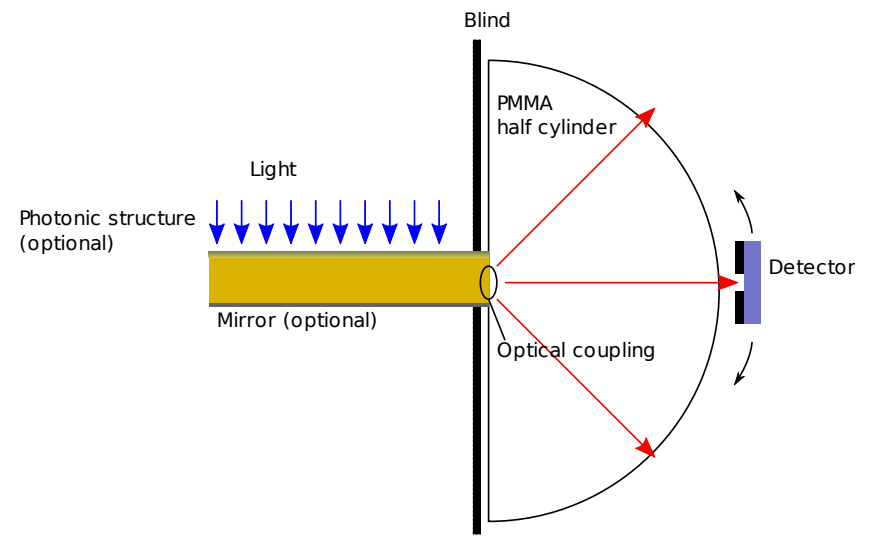

Figure 10: Experimental setup for measuring the angular distribution.

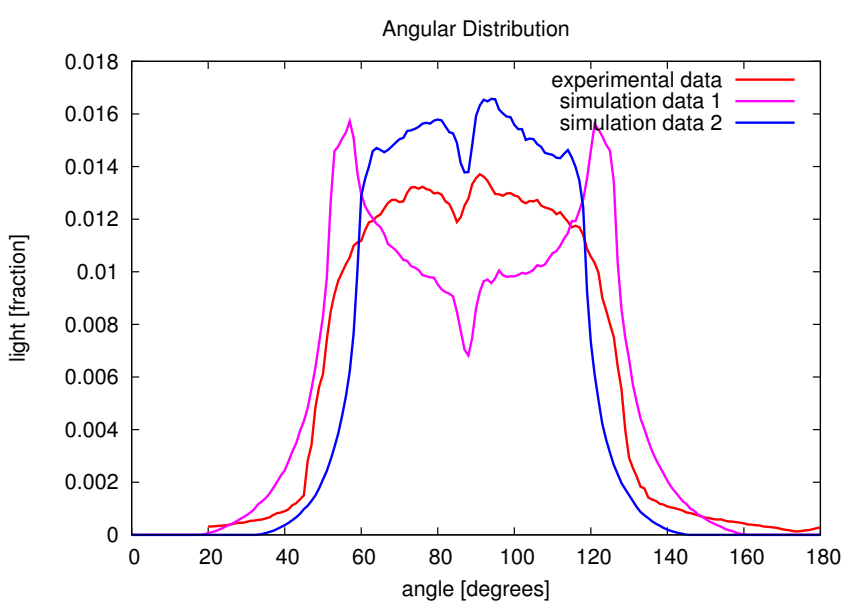

Figure 11: Fraction of light that leaves the concentrator at each angle at the rim. For details about the parameters, see Section 4.1, Angular Experiment.

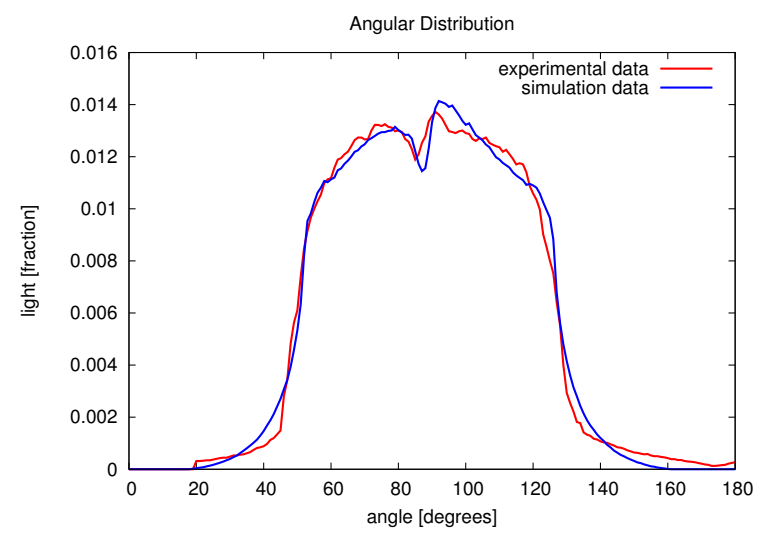

Figure 12: Result of the angular experiment simulation after parameter fitting. One parameter is twice as big as in the real experimental setup. 
integrate all light entering the sphere, which can then be measured with a single sensor. The simulation results match the experimental data pretty close, as can be seen in Figure 7. The curve of the simulation data is a bit lower as the experimental curve for wavelengths above $500 \mathrm{~nm}$ and a bit higher for lower wavelength. This might be an indicator for inaccuracies in the calculation of the absorption that was used as an input for our simulation.

Reflection Experiment The name of this experiment stems from the fact that most light detected here is due to reflection on the boundary (see Figure 6, right). The incoming light beam hits the probe with an angle of 8 degrees. The sensor will detect all light leaving the probe with the reflection angle, which also includes light being reflected at the back side of the probe. There might also be some rays that are scattered in the concentrator material (or absorbed in the dye and re-emitted) and led back to the sensor without being reflected anywhere.

The results of this experiment are shown in Figure 8. As in the transmission experiment the simulation curve is a bit higher for wavelengths lower than $500 \mathrm{~nm}$.

Absorption Using the data from the transmission and the reflection experiment the fraction of light experiencing at least one absorption event on its way through the concentrator can be calculated as

$$
\text { Absorption } \approx 1-\text { Reflection }- \text { Transmission } .
$$

In our simulation we can directly estimate the rays that had an absorption event. Figure 9 shows the comparison of the calculated absorption and the absorption in our simulation, which fits perfectly. This proves the correctness of our absorption simulation.

Angular Experiment Figure 10 shows another experimental setup which we tried to reproduce: A large PMMA half cylinder was optically coupled at one rim of the concentrator. A detector was moved in a half circle along the edge of the cylinder to measure the intensity of the light coming out of the concentrator. Thus the fraction of light that leaves the concentrator could be determined for each angle.

Figure 11 shows result for two different simulation setups: One measures simply at the desired angles at the rim. The other one considers different conditions of the experimental setup, like the inaccuracies from the fact that there is a blind where the cylinder is fixed to the concentrator and that this blind absorbs a fraction of the light that would normally be totally reflected. This fraction is not known, so we had to estimate it by a parameter variation. The different height of the peaks stems from the fact that the curves are normalised to integrate to one. The width of the peak is influenced by the ratio of the concentrator width and the cylinder radius.

This setup is quite complex and as the diagram shows our simplified model of it is not yet sufficient, but we already have a similar curve and the cosine-like shape at the top. We are even able to produce a curve that fits the experimental data quite well (see Figure 12). Unfortunately we have to set the cylinder radius on twice its real value to receive this result. We now have to extend our model in order to get better results or try to find an error in our simulation.

If the simulation setup conforms to the experimental one, we can distinguish errors and inaccuracies in the underlying model by the differences between the measured and the simulated data. This is important for a deeper understanding of the physical processes and can lead to improved models and concepts for fluorescent concentrators.

\subsection{Visualization and Renderings}

We included the measured data in an interactive, spectral rendering system (see Figure 13). The implementation is similar to adjoint photons [11], but with an additional light tracing pass, as described

\begin{tabular}{|r|r|r|r|}
\hline geometry & frames/sec & samples/sec & rays/sec \\
\hline \hline dragon (7M tris) & 3.8 & $0.5 \mathrm{M}$ & $2.4 \mathrm{M}$ \\
probe (16 tris) & 10.7 & $1.6 \mathrm{M}$ & $6.0 \mathrm{M}$ \\
\hline
\end{tabular}

Table 1: Timings for the interactive visualization of the simulation. The preview has a resolution of $480 \times 320$, and the program was run on an Intel Core 2 Quad (Q6600 @ 2.40GHz).
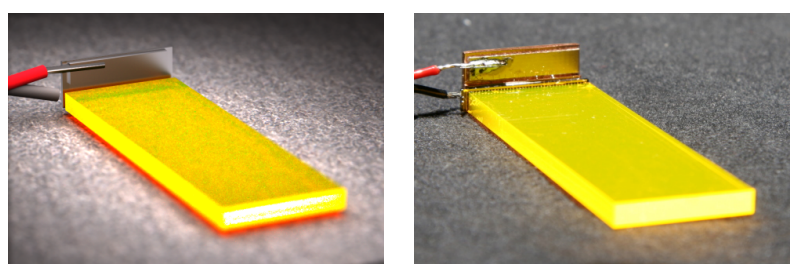

Figure 13: Comparison of a rendered probe (left) and a photograph of the real probe (right). The fact that the edges are brighter as the top is because of the light guiding effect.

above. The only difference in the adjoint transport is that the PL spectrum needs to be applied before sampling a new wavelength. Unfortunately this encumbers importance sampling by the PL spectrum, as done for the light tracing pass.

Without fluorescence, the dragon would look like the right image in Figure 14. Employing just an ordinary yellow dye would change the color of the caustics under the dragon.

Fast ray tracing allows for interactive feedback when changing parameters like lighting conditions and material properties of the fluorescent concentrator. For simple shapes, as the original probe, even interactive changes to the geometry would be possible. The visualization system only draws one sample per pixel when parameters are changed (see Figure 15), but quickly accumulates more samples to a converging image if the parameters are left unchanged. For timings, please see Table 1.

\section{CONCLUSION AND FUtURE WORK}

We developed a simple model for the simulation of a fluorescent concentrator. In the process of development we already achieved some new insights in the physical processes involved. By enabling and disabling processes, we can identify the reasons for special characteristics in the measured data. In our simulation, we can make arbitrary measurements, in contrast to real experiments which depend on the physical feasibility of the apparatus. The statistics we receive that way make it possible to develop and test enhanced concepts for fluorescent concentrators.

There are several points that can be improved in our model. We will adapt the phase function of the dye and introduce some effects which we regarded as negligible so far. Furthermore we will change the boundary conditions by adding filters, mirrors and other
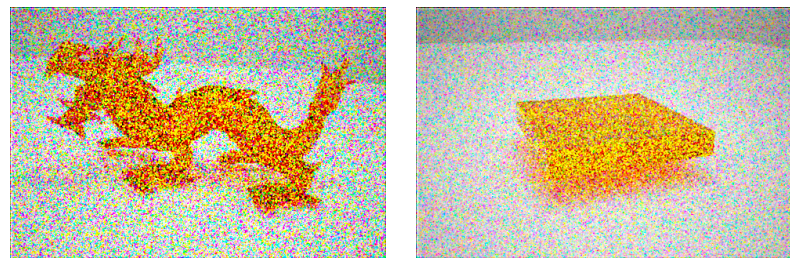

Figure 15: Interactive previews of a fluorescent dragon and the probe. 

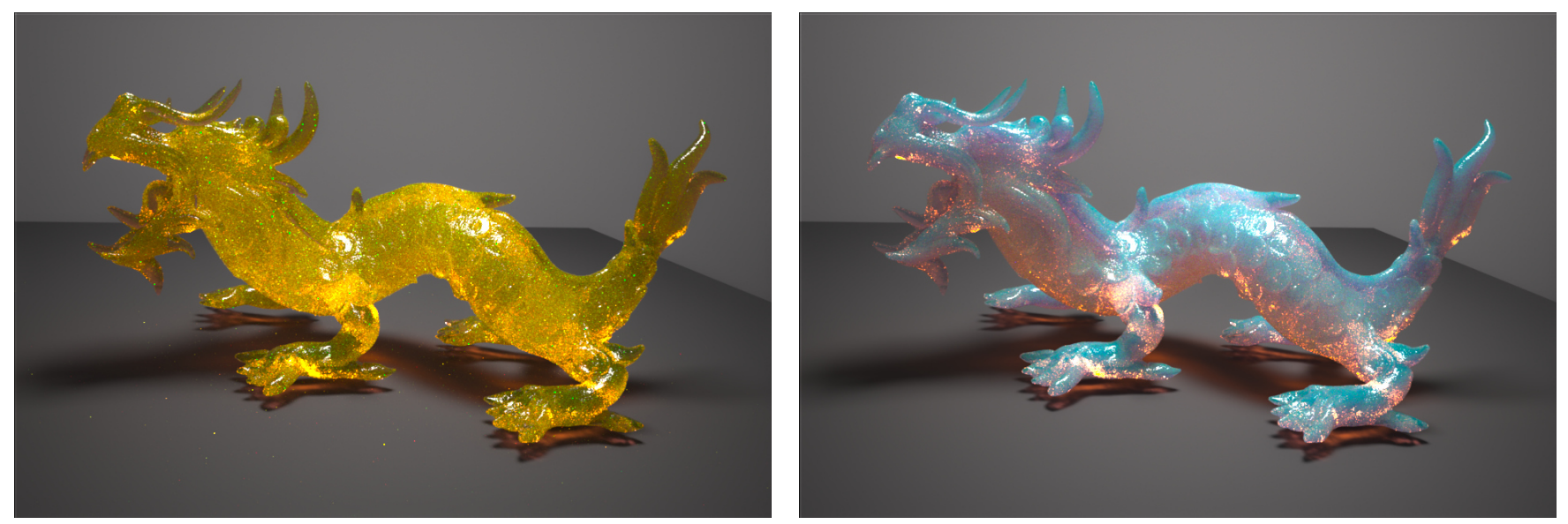

Figure 14: A dragon with fluorescent dye (left) and the same dragon rendered without fluorescence (right).

elements to the model and we will investigate the influence of optical coupling of these elements to the concentrator. In addition we will test the concept of a concentrator stack that uses different dyes and solar cells for different spectral intervals.

We deliberately chose a simple brute force approach for the simulation. This enabled easy and transparent addition and removal of simulation features. By employing fast ray tracing we could simulate more than one million paths per second despite the complexity of the underlying physics. Because of this speed it now seems feasible to develop automatic optimization or fitting algorithms. We hope, for example, to find approximations of the unknown phase function of the dye or to optimize the shape and size of the concentrator.

\section{References}

[1] American Society for Testing and Materials. Reference solar spectral irradiance: Air mass 1.5. http://rredc.nrel.gov/solar/spectra/am1.5/.

[2] A. Burgers, L. Slooff, A. Buchtemann, and J. van Roosmalen. Performance of Single Layer Luminescent Concentrators with Multiple Dyes. In Conference Record of the 2006 IEEE 4th World Conference on Photovoltaic Energy Conversion, pages 198-201, 2006.

[3] A. Burgers, L. Slooff, R. Kinderman, and J. van Roosmalen. Modelling of Luminescent Concentrators by Ray-Tracing. In Proceedings of the 20th European Photovoltaic Solar Energy Conference and Exhibition, 2005.

[4] M. Carrascosa, F. Agullo-Lopez, and S. Unamuno. Monte Carlo simulation of the performance of PMMA luminescent solar collectors. Applied Optics, 22:3236-3241, 1983.

[5] H. Dammertz, J. Hanika, and A. Keller. Shallow bounding volume hierarchies for fast SIMD ray tracing of incoherent rays. In Rendering Techniques 2008 (Proc. 19th Eurographics Symposium on Rendering), 2008. to appear.

[6] K. Devlin, A. Chalmers, A. Wilkie, and W. Purgathofer. Star: Tone reproduction and physically based spectral rendering. In State of the Art Reports, Eurographics 2002, pages 101-123. The Eurographics Association, 2002.

[7] S. Ermakow. Die Monte-Carlo-Methode und verwandte Fragen. VEB Deutscher Verlag der Wissenschaften, 1975.

[8] A. Glassner. A model for fluorescence and phosphorescence. In Proceedings of the 5th Eurographics Workshop on Rendering, 1994.

[9] J. Goldschmidt, S. Glunz, A. Gombert, and G. Willeke. Advanced fluorescent concentrators. In Proceedings of the 21st European Photovoltaic Solar Energy Conference, 2006.

[10] K. Heidler. Wirkungsgraduntersuchung zur Solarenergiekonversion mit Fluoreszenzkollektoren. PhD thesis, Albert-Ludwigs-Universität Freiburg, 1982.
[11] R. K. Morley, S. Boulos, J. Johnson, D. Edwards, P. Shirley, M. Ashikhmin, and S. Premože. Image synthesis using adjoint photons. In GI '06: Proc. of Graphics Interface 2006, pages 179-186, 2006.

[12] M. Peters, J. Goldschmidt, P. Loeper, A. Gombert, and G. Willeke. Application of photonic structures on fluorescent concentrators. In Proceedings of the 22nd European Photovoltaic Solar Energy Conference, 2007.

[13] A. Schüler, A. Kostro, C. Galande, M. Valle del Olmo, E. de Chambrier, and B.Huriet. Principles of Monte-Carlo Ray-Tracing Simulations of Quantum Dot Solar Concentrators. In Proceedings of the ISES solar world congress 2007, 2007.

[14] I. Sobol'. A Primer for the Monte Carlo Method. CRC Press, 1994.

[15] P. Susila and J. Naus. A Monte Carlo study of the chlorophyll fluorescence emission and its effects on the leaf spectral reflectance and transmittance under various conditions. Photochemical \& Photobiological Sciences, 6:894-902, 2007.

[16] A. Welch, C. Gardner, R. Richards-Kortum, E. Chan, G. Criswell, J. Pfefer, and S. Warren. Propagation of fluorescent light. Lasers in Surgery and Medicine, 21:166-178, 1997.

[17] A. Wilkie, R. Tobler, and W. Purgathofer. Combined rendering of polarization and fluorescence effects. In Proceedings of the 12th Eurographics Workshop on Rendering, 2001.

[18] A. Wilkie, A. Weidlich, C. Larboulette, and W. Purgathofer. A reflectance model for diffuse fluorescent surfaces. In Proceedings of the 4th International Conference on Computer Graphics and Interactive Techniques in Australasia and the Southeast Asia, 2006.

[19] A. Zastrow. Physikalische Analyse der Energieverlustmechanismen im Fluoreszenzkollektor. $\mathrm{PhD}$ thesis, Albert-Ludwigs-Universität Freiburg, 1981. 